\title{
PERFIL DAS FAMÍLIAS E DO ATENDIMENTO EM \\ SAÚDE PRESTADO A CRIANÇAS VÍTIMAS DE VIOLÊNCIA DOMÉSTICA NO INTERIOR DO PARANÁ
}

\author{
Sérgio Murilo Ferreira ${ }^{1}$ \\ Graduação em Psicologia \\ Faculdades Integradas \\ Pitágoras, graduação em \\ Biomedicina pela Universidade \\ de Uberaba e mestrado em \\ Promoção da saúde pelo Centro \\ de Ensino Superior de Maringá. \\ Atualmente é psicólogo da \\ Prefeitura Municipal de Ivaté. E- \\ mail: \\ srgmuriloferreira3@gmail.com
}

\section{Rute Grossi Nilani²}

Graduação em Psicologia pela Universidade Estadual de Maringá, mestrado e doutorado em Medicina (Saúde Mental) pela Universidade de São Paulo. Especialista em Saúde Coletiva pela Universidade

E-mail: rutegrossi@uol.com.br

Rose Mari Bennemann ${ }^{3}$ Graduada em Nutrição pela Universidade do Vale do Rio dos Sinos -UNISINOS, mestre e doutora em Saúde Pública, área de concentração Nutrição, pela Faculdade de Saúde Pública (FSP), Universidade de São Paulo (USP).

\author{
PROFILE OF FAMILIES AND HEALTH CARE PROVIDED TO \\ VICTIMS OF DOMESTIC VIOLENCE IN THE INTERIOR OF \\ PARANÁ
}

\section{RESUMO}

A despeito da legislação Brasileira atual, que visa proteger e garantir os direitos da criança e do adolescente, a violência doméstica ainda é uma realidade presente no país. O presente artigo buscou analisar o perfil das famílias e do atendimento em saúde prestado pelo Núcleo de apoio à Estratégia Saúde da Família (NASF) às crianças vítimas de violência doméstica no interior do Paraná. Trata-se de uma pesquisa descritiva documental, que analisou o perfil das famílias que possuem crianças vítimas de violência doméstica em seu meio e levantou a frequência de atendimentos prestados a essas crianças. Os resultados encontrados mostraram que a maior parte das famílias adota a violência como forma de resolução de problemas e que as crianças vítimas de violência raramente são identificadas nos atendimentos em saúde do município. Tais resultados indicam não só a necessidade do reforço da educação continuada para os profissionais de saúde, mas também de novas estratégias e técnicas que melhorem o desempenho dos profissionais de saúde no enfrentamento do problema.

PALAVRAS-CHAVE: Violência doméstica. Adolescente. Agressão. Negligência.

\section{ABSTRACT}

Despite the current Brazilian legislation, which aims to protect and guarantee the rights of children and teenagers, domestic violence still is a reality present in the country. This article sought to analyse the profile of families that showed cases of domestic violence identified by the Nasf of a small town in Paraná, assessing the history of violence, the characteristics of the families and pull the records of health support given to children victims of domestic violence. Is a descriptive documentary research that analyzed the profile of families who have children victims of domestic violence in their environment and raised the frequency of care given to these children. The data found shows that most of the families present a systemic state, where violence is adopted as a way to solve problems and that the children victims of violence are rarely identified during health assessment in the town. This result indicates the need to provide more training to health professionals and to elaborate new strategies to promote health in order to improve the performance of health professionals in the confrontation against this issue. 
Keywords: domestic violence, teenager, assault, negligence.

\section{INTRODUÇÃO}

A violência doméstica contra crianças pode ser definida como o ato, ou omissão, praticado pelos responsáveis ou indivíduos da convivência íntima da vítima, que gere danos de qualquer natureza (MAGALHÃES et al., 2017). Essa violência ocorre dentro de um contexto de normas sociais que difundem práticas de violência por meio de conceitos tradicionais, de modo que a violência se perpetua por meio da cultura. Em geral, a violência doméstica responde pela maior parte dos casos de violência praticada contra crianças. Tais práticas têm em geral alto grau de reincidência e frequentemente resultam em sofrimento emocional, ou mesmo físico, da vítima (APOSTÓLICO; HINO; EGRY, 2013). A criança que está submetida à constante violência apresenta, com frequência, sinais e sintomas resultantes do sofrimento que está Ihe sendo infligido, como dificuldade de aprendizado, menor tolerância à frustração, agressividade com colegas e outras pessoas, dificuldade de lidar com as próprias emoções e repetição da violência com outras crianças. Quando adultos, podem vir a apresentar dificuldades de relacionamento, com uniões frágeis e instáveis ou reproduzir o comportamento agressor (APOSTÓLICO; HINO; EGRY, 2013; HIDELBRAND et al., 2015). Achados clínicos, também, correlacionam a exposição ao sofrimento e à angústia na infância ao desenvolvimento de distúrbios mórbidos, de várias naturezas, na vida adulta, como obesidade, diabetes do tipo II, doença inflamatória intestinal, distúrbio de humor, ansiedade, problemas de sono, entre outros (PINTO JÚNIOR; CASSEP-BORGES; SANTOS, 2015, CAMPOS JÚNIOR, 2016).

Em razão da necessidade de proteção especial à criança e ao adolescente, adotou-se, na política brasileira, desde a constituição de 1988, o Estatuto da Criança e do Adolescente (ECA). O estatuto, reafirmado em 1990, institui a obrigatoriedade de notificação das situações de violência e a política de amparo e defesa das crianças e dos adolescentes, considerando o valor essencial destas como cidadãs e responsáveis pelo futuro. O ECA atua em três eixos: promoção dos direitos mediante políticas sociais básicas; defesa dos direitos, mediante intervenções de órgãos de proteção, e controle social, mediante a participação da sociedade na formulação de leis (SILVA JÚNIOR et al., 2017; BARBIANI, 2016).

Em 2011, com a publicação da Portaria 104, de 25/01/2011, foram incluídas na lista de agravos as situações de violência sexual, entre outros tipos de violência, tornando-os também de notificação obrigatória. Segundo Egry, Apostólico e Morais (2018), a notificação é um dos mais importantes instrumentos para o combate à violência infantil, pois dela derivam os demais procedimentos a serem adotados pelas redes de proteção à criança. Os autores pontuam que a notificação pode ser considerada um ato de cuidado, já que possibilita a adoção de medidas adequadas de intervenção psicossocial nas famílias e atendimentos em saúde às vítimas.

Todavia, a implementação desses princípios dentro dos lares esbarra em diversos atravessamentos. Primeiramente, é difícil coletar dados e realizar denúncias em casos que envolvam violência doméstica, o que acaba dificultando a elaboração de estimativas seguras (HIDELBRAND et al. 2015, ASSIS et al., 2012). Outra dificuldade é a cumplicidade dos envolvidos no esforço por ocultar os casos (LOBATO, MORAES, NASCIMENTO; 2012; 
PORTO; BISPO JÚNIOR; LIMA; 2014), ou mesmo pela tolerância social em relação a esse tipo de violência (PORTO; BISPO JÚNIOR; LIMA, 2014).

Diversos estudos apontam que a violência doméstica que vitima crianças e adolescentes é ainda uma realidade muito presente no Brasil. De modo geral, são mais frequentes as notificações de violência cometidas contra crianças com idade de dez anos ou mais (GAWRYSZEWSKI et al., 2012; ASSIS et al., 2012; RODRIGUES et al., 2017). Porém, acredita-se que as crianças com idade até cinco anos sejam as vítimas mais vulneráveis (NUNES e SALES, 2016; SILVA JÚNIOR et al., 2017). Em geral, o autor da violência é alguém que passa muito tempo próximo à criança. Os estudos apontam que a mãe é o agressor mais frequente, seguida do pai, do padrasto e da madrasta (GAWRYSZEWSKI et al., 2012, ASSIS et al., 2012, OLIVEIRA et al., 2011, BRANCO e TOMANIK, 2015, VALENTE et al., 2015, MATTOS DA SILVA; LIMA; LURDERMIR, 2017, MAGALHÃES et al., 2017, MAIA et al., 2017).

No tocante aos tipos de violência mais frequentes, a maior parte dos estudos aponta a negligência como a mais comum, seguida da violência física, violência sexual e, por fim, a violência psicológica (ASSIS et al., 2012; BRANCO e TOMANIK, 2015). Em geral, quase todos os estudos mostram que a maior parte das vítimas são meninas, mas discordam na diferença entre a quantidade de vítimas de cada sexo. (ASSIS et al., 2012; BRANCO e TOMANIK, 2015; GAWRYSZEWSKI et al., 2012; OLIVEIRA et al., 2011).

Concebe-se, atualmente, a violência doméstica não só como uma questão legal, mas também como um problema de saúde. A Organização Mundial da Saúde preconiza que as intervenções nessas circunstâncias se efetuem em qualquer nível, seja primário, seja secundário, seja no terciário.

A intervenção em âmbito primário tem por objetivo evitar a sua ocorrência, mediante a promoção de ações de prevenção e de cuidado (OLIVEIRA; SIMÕES, 2014). Cabe à Atenção Básica em saúde atuar ativamente na proteção da criança, identificando e intervindo do modo mais assertivo possível nas situações de violência e agravo que se estabeleçam dentro dos lares e ambientes domésticos. Compete à Atenção Básica, deste modo, promover ações de alcance individual e coletivo, que objetivem a promoção de saúde, a prevenção de agravos e o auxílio na recuperação e reabilitação. Deve ainda atuar com uma perspectiva ampla em saúde, que contemple o cidadão em todos determinantes biológicos, psicológicos e sociais (KANNO; BELLODI; TESS, 2012). Tal caráter confere à Atenção Básica um importante potencial no enfrentamento da violência contra crianças, haja vista que o cuidado integral aos usuários do serviço de saúde valoriza a formação de um sólido vínculo com as comunidades a serem atendidas. Por meio de estratégias de aproximação, são identificadas as demandas e dificuldades enfrentadas pela comunidade atendida (KANNO; BELODI; TESS, 2012; PORTO; BISPO JÚNIOR; LIMA, 2014).

A Estratégia de Saúde da Família (ESF), como porta de entrada preferencial da Atenção Básica, é fundamentada na abordagem coletiva, multi e interprofissional, centrada na família e na comunidade. A propósito, é composta por equipes da saúde da família que envolvem enfermeiros, médicos, técnicos em enfermagem, odontólogos e Agentes Comunitários de Saúde (ACS). Os ACS, particularmente, no papel de interlocutores entre a comunidade e os serviços de saúde, abrem amplas possibilidades de identificação e de intervenção em situações de violência doméstica contra crianças (MOREIRA et al., 2014). Não obstante, existem também obstáculos à eficácia dos trabalhos das equipes da ESF no combate à violência doméstica. Primeiramente, se a proximidade do Agente Comunitário de Saúde com a população facilita o acesso 
às famílias, do mesmo tempo pode comprometer o processo de cuidar, pois obriga a equipe a agir com cautela diante de situações delicadas, uma vez que esses profissionais estão também expostos a possíveis represálias caso reportem alguma situação de violência à equipe de saúde. Muitas vezes, situações de violência frequente dentro de um lar coexistem com práticas ilegais e envolvimento com crimes por parte de um ou mais membros da família, o que intimida muitos dos agentes de saúde (KANNO; BELODI; TESS, 2012; EGRY et al., 2017, MOREIRA et al., 2014; PORTO; BISPO JÚNIOR; LIMA, 2014; LOBATO, MORAES, NASCIMENTO, 2012; OLIVEIRA et al., 2015; DAIANE SILVA et al., 2015; RODRIGUES et al., 2017).

A literatura também aponta a falta de preparo dos profissionais da saúde para lidar com situações de violência intrafamiliar, denunciando a falta de conhecimento destes sobre o assunto (KANNO; BELODI; TESS, 2012; MOREIRA et al., 2014; PORTO; BISPO JÚNIOR; LIMA, 2014, LOBATO; MORAES; NASCIMENTO, 2012; OLIVEIRA et al., 2015; PINTO JÚNIOR; CASSEP-BORGES; SANTOS, 2015; EGRY et al., 2017).

Da mesma forma, a atuação dos profissionais da Atenção Básica em saúde, no enfrentamento da violência doméstica contra crianças, esbarra na complacência ou mesmo na omissão dos profissionais frente a situações mais sutis de violência, que, com frequência, são naturalizadas na sociedade como práticas tradicionais de educação (KANNO; BELODI; TESS, 2012; PINTO JÚNIOR; CASSEP-BORGES; SANTOS, 2015, BARBIANI, 2016).

O Núcleo de Apoio à Estratégia Saúde da Família (doravante NASF), dada a sua natureza interdisciplinar, tem notável potencial para promover o enfrentamento desse problema, seja pelo apoio e orientação que fornece às outras equipes de saúde, seja mediante o estabelecimento de um Projeto Terapêutico Singular quando necessário (GOIS DOS SANTOS; UCHÔAFIGUEIREDO; LIMA, 2017).

Os Núcleos de Apoio à Saúde da Família foram criados em janeiro de 2008, com a proposta de fornecer apoio interdisciplinar às equipes de Atenção Básica e estender as possibilidades de ofertas de cuidado aos usuários (GÓIS DOS SANTOS; UCHÔA-FIGUEIREDO; LIMA, 2017). Ainda que o Agente Comunitário de Saúde (ACS) tenha um papel fundamental na atuação contra a violência doméstica, a literatura aponta a importante atuação das equipes do NASF no apoio, na orientação e na resolução de casos identificados de violência (MOREIRA et al., 2014).

\section{MATERIAL E MÉTODOS}

Trata-se de uma pesquisa documental quantitativa desenvolvida mediante a aprovação do Comitê de Ética em Pesquisa, sob o parecer 2022086. A pesquisa foi realizada no NASF, em um município do interior do Paraná, localizado a 644 quilômetros da capital Curitiba. Esse município conta com uma população aproximada de 9.512 habitantes (Brasil, IBGE, 2016). O NASF local dispõe de uma equipe composta por três profissionais: psicólogo, fisioterapeuta e nutricionista. Tais profissionais atuam de forma interdisciplinar na avaliação, no encaminhamento e no atendimento dos casos recebidos. Também são realizadas intervenções em parceria com outros órgãos, como a Atenção Básica em saúde e os serviços de assistência social.

Foram consultados os registros de atendimentos das crianças, após autorização da Secretaria Municipal de Saúde. O pesquisador também se comprometeu a 
preservar a privacidade das informações dos pacientes com a assinatura de um termo de confidencialidade.

Selecionaram-se, como período para consulta, os anos de 2015 e 2016. Foi realizado levantamento dos casos de crianças e adolescentes que apresentavam histórico de risco psicossocial associado à violência doméstica atendido pelo setor de Psicologia. Esses prontuários oferecem informações a respeito do paciente e têm o objetivo de organizar os procedimentos relacionados ao acompanhamento psicológico. Classificaram-se como violência doméstica situações em que havia agressão física à criança, negligência e abandono por um ou ambos os pais, situações em que a criança estava exposta a ameaças ou a agressões verbais e suspeita de violência sexual.

Com vistas a verificar o histórico de saúde das crianças identificadas por meio do levantamento no NASF, foram consultados os prontuários delas no Serviço de Saúde do Município e no sistema digital online e-SUS, referentes aos últimos seis anos, incluindo consultas de rotina e de pronto atendimento, com o intento de verificar queixas, diagnóstico e número de atendimentos prestados a cada ano. Em posse dos registros, deu-se seguimento à análise documental. Segundo Bardin (2010), a análise documental pode ser assim definida:

"[...] uma operação ou um conjunto de operações visando representar o conteúdo de um documento sob uma forma diferente do original, a fim de facilitar, num estado ulterior, a sua consulta e referenciação" (CHAUMIERC (1974), in BARDIN, 2010, p. 47).

Ao fim da fase preliminar, deu-se segmento à fase mais extensa, de exploração do material a ser analisado, na qual foram aplicadas operações de codificação: a pré-análise, a exploração do material e a inferência e interpretação dos dados; a aplicação sistemática dos procedimentos de análise adotados, em que se construiu o processo de análise para verificar, a partir da pesquisa, quais outras demandas em saúde mais frequentemente acometem crianças identificadas como vítimas de violência doméstica, conforme proposto por Bardin (2010).

\section{RESULTADOS E DISCUSSÃO}

Entre o início do ano de 2015 e fim do ano de 2016, foram identificados 39 registros de vulnerabilidade associada à violência doméstica contra crianças e adolescentes. Entre os casos, 22 vítimas eram meninas e 17 eram meninos. Dos casos identificados, apenas 1 era contra uma criança com menos de cinco anos; 21 casos foram identificados com crianças na faixa de idade dos seis aos dez anos de idade, e os demais casos foram identificados em adolescentes (12 anos de idade ou mais). 
Tabela 1: Distribuição das vítimas, segundo sexo e grupo etário.

\begin{tabular}{llll}
\hline & Vítimas & N & $\%$ \\
\hline Sexo & Feminino & 22 & 56 \\
& Masculino & 17 & 44 \\
Grupo & Menos de 5 anos & 1 & 2 \\
etário & & & \\
& Entre 5 e 11 anos de idade & 21 & 54 \\
& Acima de 12 anos de idade & 14 & 35 \\
\hline
\end{tabular}

Na maioria dos casos, a mãe foi a principal responsável pela agressão sofrida pelas crianças, em especial pela agressão contra meninas. Ressalta-se, entretanto, que, em grande parte dos casos, havia mais de um agressor em casa, de modo que, ainda que mais comumente a mãe fosse a principal responsável pela agressão, o pai, padrasto ou outros parentes que vivessem na mesma casa eram também responsáveis pela situação.

Dos 39 casos identificados de violência doméstica contra crianças, a mãe era ou participante ou a principal responsável por $64,1 \%, 25$ deles; o pai era 0 principal responsável ou participante em $51,3 \%, 20$ das situações; tios e outros parentes mais distantes participaram de 18\%, 7 das ocasiões; irmãos participaram em 12,6\%, 5 dos casos; padrastos, em 10,3\%, 4 dos eventos; e avós/avôs, em 5,1\%, 2 das ocasiões.

Tabela 2: Caracterização dos agressores em frequência ( $F)$ e porcentagem (\%)

\begin{tabular}{llll}
\hline & Caracterização do agressor & F & $\%$ \\
\hline Agressor & Mãe & 25 & 64 \\
& Pai & 20 & 51 \\
& Parente mais distante (tio, primo) & 7 & 18 \\
& Irmão & 5 & 13 \\
& Padrasto & 4 & 10 \\
& Avô/Avó & 2 & 05 \\
Adversidades & Mais de um agressor & & \\
associadas & Ocorrência de violência interparental & 24 & 62 \\
na família & Histórico de envolvimento com crimes & 12 & 31 \\
& Histórico de doença mental crônica ou uso de & 11 & 28 \\
& álcool e/ou outras substâncias psicoativas & & \\
\hline & & &
\end{tabular}


Em doze dos casos identificados, verificou-se na família histórico de envolvimento com atividades criminosas e prisão de parentes próximos do menor identificado como vítima. Em $28,2 \%$ dos fatos, havia caso de doença mental crônica ou de uso crônico de substâncias psicoativas em algum dos parentes próximos que viviam na mesma casa da criança. Casos como o descrito a seguir ilustram o contexto de violência que, com frequência, acometem crianças:

VML, sexo feminino (11 anos), que morava com a mãe, o padrasto e a irmã (4 anos), esta filha de seu padrasto e de sua mãe. VML viveu com a avó materna até os oito anos de idade, quando foi para a casa da mãe, que, nesta época, já era casada com o padrasto. Logo nos primeiros meses, VML foi obrigada a assumir todas as tarefas de casa, a limpeza das roupas, da louça, entre outras atividades, e tinha praticamente todo o seu tempo fora da escola ocupado com esses serviços. A mãe vistoriava minuciosamente a casa quando chegava do trabalho, na expectativa de encontrar algum motivo para punir a menina, às vezes se irritava quando não o encontrava. A mãe também incentivava a irmã pequena de VML a denunciar a garota, e, caso está o fizesse, VML era punida sem nenhum direito a se justificar. As punições eram sempre físicas. O padrasto cooperava na manutenção da situação, sempre no argumento de que faziam isso para o bem da própria VML. O padrasto começou a procurar mais contato físico com a menina, sempre com justificativas vagas. Em uma ocasião, ele pediu para que a menina erguesse a saia após uma surra aplicada pela mãe, sob o pretexto de que queria conferir seus vergões. Posteriormente, ordenou que a menina lhe mostrasse as suas partes íntimas, pois queria conferir se ela não tinha "perdido a sua virgindade". Os professores, ao perceberem a mudança gradativa no comportamento de VML, solicitaram a parceria do serviço de proteção à criança do município (CREAS) e da Atenção Básica em saúde. Após averiguação, foi confirmada a situação de exposição à violência doméstica, então foram adotadas as devidas providências legais (retirada da guarda da mãe e concessão da guarda à tia materna, mas permitindo acesso irrestrito da mãe para entrar em contato com a filha).

Entre os casos detectados, $61,5 \%$, 24, das crianças sofreram mais de um tipo de violência. Quanto ao tipo, 48,7\% (19), foram violência física; em 46,1\% (18), ocorreu violência psicológica; em 43,5\% (17), foi constatada negligência por parte dos pais; e em 7,7\% (3) foram confirmadas situações de violência sexual. Os casos de negligência são associados comumente à alienação de um ou de ambos os pais.

Tabela 3: Caracterização do tipo de violência em frequência ( $F)$ e porcentagem

$(\%)$

\begin{tabular}{|c|c|c|c|}
\hline & Caracterização da violência & $F$ & $\%$ \\
\hline Tipo & Violência física & 19 & 48,7 \\
\hline \multirow[t]{3}{*}{ violência } & Negligência & 17 & 43,5 \\
\hline & Violência psicológica & 10 & 25,6 \\
\hline & Violência sexual & 3 & 07,7 \\
\hline
\end{tabular}

Normalmente, a identificação dos casos de violência doméstica contra crianças é mediada pela parceria com outros órgãos do município e de outros entes federativos (Estado, União) que atuam no município, como a Escola Estadual 
Rachel de Queiroz e a polícia civil. Nos episódios de violência confirmados, 35,9 foram constatados pelas escolas municipal e estadual; $12,8 \%$ vieram encaminhados pelos órgãos de serviço social do município (CRAS, CREAS); $12,8 \%$ foram dirigidas ao NASF por pedido médico; $2,6 \%$ vieram a pedido da polícia civil; e 2,6\% foram atendidas por ordem judicial da comarca de Icaraíma. Nenhuma das situações foi identificada pelo trabalho das Agentes Comunitárias de Saúde.

Tabela 4: Órgãos parceiros em frequência (F) e porcentagem (\%)

\begin{tabular}{lll}
\hline Órgãos notificador & F & $\%$ \\
\hline Escola & 27 & 69,2 \\
Serviço social & 5 & 12,8 \\
Serviço de saúde & 5 & 12,8 \\
Polícia civil & 1 & 02,6 \\
Promotoria & 1 & 02,6 \\
\hline
\end{tabular}

Nos registros da Unidade Básica de Saúde e do sistema digital on-line e-SUS dos últimos seis anos, 39 crianças e adolescentes foram selecionados. A investigação dos registros de atendimentos em saúde prestados evidenciou 0 desconhecimento por parte dos profissionais da situação de vulnerabilidade. Em geral, salvo algumas exceções, perceberam-se poucos registros de atendimento médico dessas crianças. Das 39 vítimas de violência doméstica, 43,6\% possuem uma média de menos de um registro de atendimento por ano; 20,5\% apresentam uma média de dois registros de atendimento em saúde por ano. Quatro crianças têm uma média de quatro atendimentos registrados por ano; outras quatro possuem uma média de três atendimentos; e três crianças possuem uma média de um atendimento. No entanto, três crianças são atendidas mais de seis vezes por ano.

Tabela 5: Atendimentos prestados às crianças e adolescentes em frequência $(F)$ e porcentagem (\%)

\begin{tabular}{lll}
\hline Frequência de atendimento & F & $\%$ \\
\hline Média de menos de um registro de atendimento por ano & 17 & 44 \\
Média de um registro de atendimento por ano & 3 & 08 \\
Média de dois registros de atendimento por ano & 7 & 18 \\
Média de três ou mais registros de atendimentos por ano & 12 & 30 \\
\hline
\end{tabular}

As queixas mais comuns nos atendimentos foram problemas respiratórios $(27,8 \%)$, gripe/febre $(25,6 \%)$, problemas gastrintestinais $(8,3 \%)$ e acidentes $(6,7 \%)$. A categoria acidentes trata-se de uma classificação ampla, pois abarca tanto queimaduras por contato com suco de limão, bem como torções nos braços e ferimentos no corpo.

Cumpre esclarecer que houve poucos casos de confirmação de anemia, obesidade e problemas de comportamento, chegando a apenas $2 \%$ cada um. Dois casos que chamam a atenção são o de duas crianças, que não tinham 
relação de parentesco entre si, e que foram atendidas mais de seis vezes por ano no município por sofrerem de problemas respiratórios crônicos, com crises recorrentes de asma e falta de ar. Na investigação do NASF, no ano de 2016, descobriu-se que os pais eram usuários crônicos de maconha.

Os achados desta pesquisa possuem grande correspondência com os achados na pesquisa bibliográfica sobre o assunto. Em consonância com a literatura, a maior parte dos casos identificados de violência doméstica praticada contra crianças corresponde a crianças que possuem mais de dez anos, as quais estão mais dispostas a questionar a violência que sofrem (GAWRYSZEWSKI et al., 2012; ASSIS et al. 2012, RODRIGUES et al., 2017). Do mesmo modo, percebese que os pais são mais comumente os autores da violência (ASSIS et al., 2012; BRANCO e TOMANIK, 2015; VALENTE et al., 2015, MAGALHÃES et al., 2016, MAIA et al., 2017). Nota-se, ainda, uma prevalência maior de casos de violência aplicada contra meninas em relação às agressões aplicadas contra meninos (ASSIS et al., 2012; OLIVEIRA et al., 2011; BRANCO e TOMANIK, 2015).

Depreende-se que o atendimento em saúde tem uma evidente dificuldade em identificar os casos de violência contra crianças, uma vez que não existe registro ou notificação por parte dos profissionais de saúde, em especial dos médicos, de ocorrência de violência doméstica. Ao longo de seis anos, houve tão somente três solicitações de atendimento em psicoterapia.

Silva Júnior et al. (2017), ao analisarem os registros de notificação de maustratos de 227 médicos de Estratégia de Saúde da Família do Ceará, averiguaram que o número esperado de notificações de maus-tratos estava aquém do esperado, sendo que muitas situações de violência contra crianças passavam despercebidas ou simplesmente eram negligenciadas.

Lima et al. (2011) apontam que a formação predominantemente clínica dos profissionais em saúde pode comprometer a habilidade destes em identificar circunstâncias de violência doméstica em crianças, uma vez que nem sempre a vitimização cursa com sinais clínicos. Aparentemente, a vivência do profissional de saúde está relacionada com sua capacidade em identificar casos de violência doméstica. A propósito, segundo diversos autores, profissionais de saúde que atuam na profissão há mais tempo tendem a notificar agravos contra crianças com mais frequência (SILVA JÚNIOR et al., 2017, LIMA et al., 2011, BANNWART; BRINO, 2011).

Bannwart e Brino (2011) apresentam, também, diversos atravessamentos na identificação por médicos pediatras de casos de violência doméstica. Entre as dificuldades, está o medo dos profissionais em se envolver legalmente com tais casos, a falta de capacitação dos profissionais para reconhecer casos de violência, a descrença de alguns profissionais no potencial dos órgãos de proteção em resolver o problema e a falta de um protocolo claro para orientar o profissional. Egry, Apostólico e Moraes, 2018 defendem que um protocolo de notificação é de fundamental importância para orientar o profissional de saúde na resolução desses casos, levando a denúncia de modo mais célere ao órgão de proteção responsável.

Cabe mencionar que, na realidade do município estudado, a atuação dos profissionais de saúde, em especial dos médicos, apresentou notificação aquém do esperado. Todavia, os profissionais de saúde também tiveram pouco contato com a maioria das vítimas. Nesse sentido, atividades em promoção de saúde, como campanhas promovidas pelo NASF, podem oportunizar o contato destes profissionais com as crianças vitimadas pela violência. 


\section{CONSIDERAÇÕES FINAIS}

O acompanhamento das famílias, por meio do qual se identificaram situações de violência doméstica no município em estudo, confirmou achados já descritos na literatura científica. Em geral, a principal responsável pela violência é a mãe, seguida de pai, tios, irmãos, padrastos e avós. Pode-se observar particularidades no presente estudo, por exemplo a identificação de que, em situações de violência doméstica, normalmente não existe só um agressor, mas dois ou mais agressores dentro da família, que atuam motivados por razões distintas. Em regra, a violência aparece como um meio de resolução de conflitos. Ao se avaliarem os registros de atendimento, observa-se que a maior parte das crianças identificadas como vítimas de violência doméstica frequenta pouco os estabelecimentos de saúde, o que compromete o potencial dos profissionais de saúde em identificar a situação de sofrimento delas. O que fica mais evidente é que a maior parte das crianças identificadas chegou ao conhecimento do NASF por intermédio das escolas.

$\mathrm{Na}$ verdade, é possível que a falta de preparo dos profissionais e de protocolos claros de procedimento para reportar tais casos tenha também se tornado atravessamentos ao enfrentamento da violência contra crianças, de modo que é desejável fornecer aos profissionais a devida qualificação para essa demanda. Grosso modo, é necessário ressaltar o papel da Atenção Básica em saúde na proteção das crianças. Isso porque sanar um ambiente familiar tomado por uma conduta de violência faz parte do cuidar, além disso as crianças são também cidadãs, detentoras de direitos e que conduzirão nossa sociedade no futuro. Ou seja, urge que se enfrente o ciclo de violência

\section{REFERÊNCIAS}

APOSTÓLICO, Maíra Rosa; HINO, Paula; EGRY, Emiko Yoshikawa. As possibilidades de enfrentamento da violência infantil na consulta de enfermagem sistematizada. Revista da Escola de Enfermagem da USP, São Paulo, v. 47, n. 2, p. 320-327, 2013.

ASSIS, Simone Gonçalves de et al. Notificações de violência doméstica, sexual e outras violências contra crianças no Brasil. Ciênc. saúde coletiva, Rio de Janeiro, v. 17, n. 9, p. 2305-2317, 2012.

BARBIANI, Rosangela. Violação de direitos de crianças e adolescentes no Brasil: interfaces com a política de saúde. ENSAIO • Saúde debate 40 (109). Rio de Janeiro, v. 40, n. 109, p. 200-211, June 2016.

BARDIN, Laurence. Análise de conteúdo. 4. edição, Lisboa: Edições 70, 2010.

BANNWART, Thais Helena; BRINO, Rachel de Faria. Dificuldades enfrentadas para identificar e notificar casos de maus-tratos contra crianças e/ou adolescentes sob a óptica de médicos pediatras. Rev. paul. pediatr., São Paulo, v. 29, n. 2, p. 138-145, 2011.

BRANCO, Marco Antônio de Oliveira; TOMANIK, Eduardo Augusto. Violência doméstica contra crianças e adolescentes: prevenção e enfrentamento.

Revista Psicologia \& Sociedade, Florianópolis, v. 24, n. 2, p. 402-411, 2012. 
CAMPOS JÚNIOR, Dioclécio. The formation of citizens: the pediatrician's role. Jornal de pediatria, Rio de Janeiro, v. 92, n. 3, p. S23-S29, 2016.

DAIANE SILVA, Camila; GOMES, Vera Lúcia de Oliveira; OLIVEIRA, Denize Cristina de; MARQUES, Sérgio Corrêa; FONSECA, Adriana Dora; MARTINS, Cibele da rocha. Representação social da violência doméstica contra a mulher entre Técnicos de Enfermagem e Agentes Comunitários. Revista Escola de Enfermagem. São Paulo, 49 (1), p. 22-29, 2015.

EGRY, Emiko Yoshikawa; APOSTOLICO, Maíra Rosa; MORAIS, Teresa Christine Pereira. Notificação da violência infantil, fluxos de atenção e processo de trabalho dos profissionais da Atenção Primária em Saúde. Ciênc. saúde coletiva, Rio de Janeiro, v. 23, n. 1, p. 83-92, 2018.

EGRY, Emiko Yoshikawa et al. Enfrentar a violência infantil na Atenção Básica: como os profissionais percebem? Rev. Bras. Enferm., Brasília, v. 70, n. 1, p. 119-125, 2017.

GAWRYSZEWSKI, Vilma Pinheiro et al. Maus-tratos contra a criança e o adolescente no Estado de São Paulo, 2009. Revista da Associação Médica Brasileira (English Edition), v. 58, n. 6, p. 659-665, 2012.

GOIS DOS SANTOS, Rosimeire Aparecida Bezerra de; UCHÔAFIGUEIREDO, Lúcia da Rocha; LIMA, Laura Câmara. Apoio matricial e ações na atenção primária: experiência de profissionais de ESF e Nasf. SAÚDE DEBATE | Rio de Janeiro, V. 41, N. 114, P. 694-706, jul-set, 2017.

HILDEBRAND, Natália Amaral et al. Violência doméstica e risco para problemas de saúde mental em crianças e adolescentes. Psicologia: Reflexão e Crítica, Porto Alegre, v. 28, n. 2, p. 213-221, 2015.

IBGE from Paraná: banco de dados disponível em <http://ibge.gov.br/cidadesat/painel/historico.php?codmun=411155\&search=par ana\%7Civate\%7Cinphographics:-history\&lang=_ES> acesso em: 24 out. 2016.

KANNO, Natália de Paula; BELLODI, Patrícia Lacerda; TESS, Beatriz Helena. Profissionais da Estratégia Saúde da Família diante de demandas médicosociais: dificuldades e estratégias de enfrentamento. Saúde e Sociedade, São Paulo, v. 21, n. 4, p. 884-894, 2012.

LIMA, Maria do Carmo Campos Santos et al. Atuação profissional da atenção básica de saúde face à identificação e notificação da violência infanto-juvenil. Revista Baiana de Saúde Pública. Salvador, v.35, supl.1, p.118-137, 2011.

LOBATO, Geórgia Rosa; MORAES, Claudia Leite; NASCIMENTO, Marilene Cabral do. Desafios da atenção à violência doméstica contra crianças e adolescentes no Programa Saúde da Família em cidade de médio porte do Estado do Rio de Janeiro, Brasil. Caderno de Saúde Pública, Rio de Janeiro, v. 28, n. 9, p. 1749-1758, 2012. 
MAGALHÃES, Júlia Renata Fernandes de, et al. Violência intrafamiliar: vivências e percepções de adolescentes. Escola Anna Nery Revista de Enfermagem, 21, 2017.

MAIA, Rosely Cardoso et al. Da Proteção ao Risco: Configurações da Violência Intrafamiliar na Juventude Paraense. Psicologia: Teoria e Pesquisa, Brasília, Vol. 33 p. 1-8, 2017.

MATTOS DA SILVA, Josiane Maria; LIMA, Marília de Carvalho; LURDEMIR, Ana Bernarda. Violência por Parceiro Íntimo e Prática Educativa Materna. Revista de Saúde Pública. São Paulo, Vol.: 51:34, p. 1-11, 2017.

MOREIRA, Tatiana das Neves Fraga et al. A construção do cuidado: o atendimento às situações de violência doméstica por equipes de Saúde da Família. Saúde e Sociedade, São Paulo, v. 23, n. 3, p. 814-827, 2014.

NUNES, Antonio Jakeulmo; VITORINO SALES, Magda Coeli. Violência Contra Crianças no Cenário Brasileiro. Revista Ciência \& Saúde Coletiva. Rio de Janeiro, Vol. 21, Issue 3, p. 871-880, 2016.

OLIVEIRA, Adriane Maria Netto de et al. percepção dos profissionais de saúde frente às intervenções primárias: prevenindo a violência intrafamiliar. Texto e contexto Enfermagem. Florianópolis, Abr-Jun, 24(2), p. 424 - 31, 2015.

OLIVEIRA, Marluce Tavares de et al. Sub-registro da violência doméstica em adolescentes: A (in) visibilidade na demanda ambulatorial de um serviço de saúde no Recife-PE, Brasil. Rev. bras. saúde matern. infant, Boa Vista, v. 11, n. 1, p. 29-39, 2011.

OLIVEIRA, Paula Sameira de, SIMOES, Ainda. Maus-tratos à infância: as referências dos técnicos das Comissões de Proteção de Crianças e Jovens. [CPCJ]. Revista Portuguesa de Enfermagem de Saúde Mental, Porto, n SPE1, p. 82-89, 2014.

PINTO JÚNIOR, Antônio Augusto, CASSEP-BORGES, Vicente, SANTOS, Janielly Gonçalves dos. Caracterização da violência contra crianças e adolescentes e as estratégias interventivas em um município do estado do Rio de Janeiro. Caderno de Saúde Coletiva, Rio de Janeiro, Vol.: 33(2), p. 124131, 2015.

PORTO, Roberta Taynan Souza; BISPO JÚNIOR, José Patrício, Lima, Elvira Caires de. Violência doméstica sexual no âmbito da estratégia de saúde da família: atuação profissional e barreiras para o enfrentamento. Physis Revista de Saúde Coletiva, Rio de Janeiro, Vol. 24(3), p. 787-807, 2014.

RODRIGUES, Nádia Cristina Pinheiro et al. The increase in domestic violence in Brazil from 2009-2014. Ciência e Saúde Coletiva. Rio de Janeiro, Vol. 22, n.9, p.2873-2880, 2017.

SILVA JUNIOR, Geraldo Bezerra da et al. Identificação e notificação de maustratos em crianças e adolescentes por médicos de família no Ceará. Trab. educ. saúde, Rio de Janeiro, v. 15, n. 2, p. 469-484, ago. 2017. 


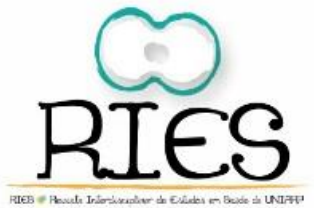

VALENTE, Leidielly Aline et al. Domestic violence against children and adolescents: prevalence of physical injuries in a southern Brazilian metropolis. Brazilian dental journal, Ribeirão Preto, v. 26, n. 1, p. 55-60, 2015.

Recebido em: 26-04-2018

Aceito em: 01-07-2019 